\title{
An Efficient Full-Wave Simulation Algorithm for Multiple Vertical Conductors in Printed Circuits
}

\author{
Tayyar Onal, M. I. Aksun, Senior Member, IEEE, and Noyan Kinayman, Member, IEEE
}

\begin{abstract}
An efficient and rigorous numerical method, based on the spatial-domain method of moments (MoM) in conjunction with the closed-form Green's functions, is devised for the analysis of multiple vertical conductors in printed circuits. As this combination has already proven to be very efficient for the analysis of printed structures with horizontal and vertical conductors, it is extended to efficiently handle multiple vertical conductors. Some circuits with multiple vertical strips are analyzed using the proposed method, and results are compared either to those presented in the literature or to those obtained from the commercial software $e m$ by SONNET Software, North Syracuse, NY. Computational efficiency of the algorithm is assessed in terms of CPU time, and it is observed that the computational cost of the proposed algorithm is an order of magnitude less than that of the straightforward implementation of the underlaying method of the algorithm-the spatial-domain MOM using closed-form Green's functions.
\end{abstract}

Index Terms-Closed-form Green's functions, discrete complex image method, method of moments (MoM), three-dimensional (3-D) planar layered media, vertical conductors.

\section{INTRODUCTION}

D URING THE last three decades, the use of printed geometries has been increasing steadily, especially in the applications of antennas and monolithic microwave integrated circuits [1]-[3]. Since circuits distributed over a multilayer environment need vertical conductors to facilitate the connectivity between the layers, vertical conductors like via-holes in monolithic microwave integrated circuits, shorting strips, and probe feeds in microstrip antennas have become integral parts of highfrequency circuits and/or multifunction antennas [4]. As a result, with the use of vertical conductors, one of the main advantages of printed circuits-ease to manufacture-might have been sacrificed to achieve the goals of multifunction operations and/or miniaturization. This burden can partially be alleviated with a computer-aided design (CAD) tool that can accurately and efficiently analyze such circuits. Therefore, parallel to such fabrication requirements of real circuits, simulation tools need to be developed to efficiently and accurately design printed circuits with multiple vertical conductors.

It is widely accepted that method-of-moments (MoM)-based algorithms are the most suitable and efficient algorithms for the solution of mixed-potential integral equations [5]-[8]. In addition, with the introduction of closed-form spatial-domain

Manuscript received March 22, 2006; revised May 31, 2006.

T. Onal and M. I. Aksun are with the Department of Electrical and Electronics Engineering, Koç University, 34450 Istanbul, Turkey (e-mail: iaksun@ku.edu. tr).

N. Kinayman is with M/A-COM, Lowell, MA 01853 USA (e-mail: kinayman@tycoelectronics.com).

Digital Object Identifier 10.1109/TMTT.2006.881616

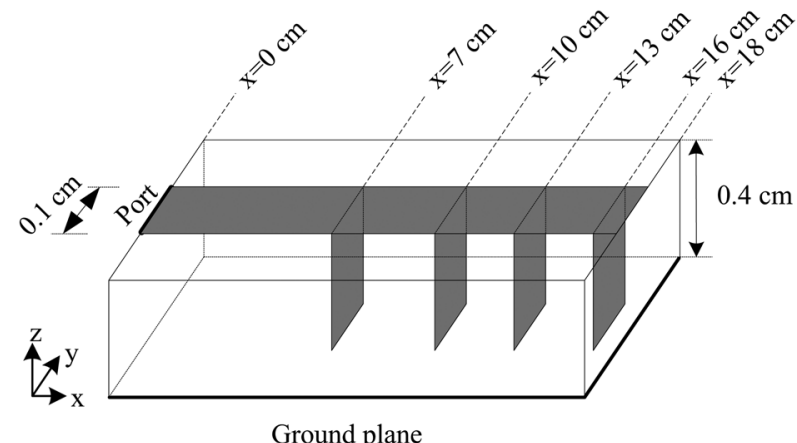

Fig. 1. Microstrip line with multiple shorting strips over a perfect electric conductor (PEC)-backed substrate $\left(\epsilon_{r}=4.0\right)$.

Green's functions for vector and scalar potentials (the procedure is also known as the discrete complex image method) [9], [10], and with its improved versions [11], the computational efficiency of the spatial-domain MoM for printed geometries has been significantly improved [12], [13]. Therefore, the main numerical technique employed in this study is chosen to be the spatial-domain MoM in conjunction with the closed-form Green's functions. A formulation how to handle vertical conductors within this approach was first proposed in [14], and extended and applied to more general geometries in [17]. Meanwhile, applications of the combination of the MoM and discrete complex image method to vertical probes and to probe-fed patch antennas in layered media were proposed following a different approach [15], [16]. In search of an efficient approach, based on a similar combination, for the analysis of general printed structures, there has recently been some significant contributions [18]-[21]. However, there has been no study thus far on the rigorous and efficient analysis of printed structures with multiple vertical conductors. The goal in this study is to develop a full-wave method that efficiently handles multiple vertical conductors in printed structures.

Since the main goal in this paper is to develop an algorithm that substantially improves the MoM matrix filling time when multiple vertical conductors are involved, the proposed algorithm, together with its formulation and demonstration, is detailed in Section II. It is then followed by some representative examples in Section III to demonstrate the efficiency of the method. Finally, in Section IV, conclusions are provided.

\section{Discussions on FILling MoM Matrix ENTRIES FOR VERTICAL CONDUCTORS}

\section{A. Formulation}

Let us consider a printed structure, as shown in Fig. 1, that supports only $x$ - and $z$-components of the current density to demonstrate the formulation. Note that, throughout this study, 
vertical strips are employed as vertical conductors, with no loss of generality, and that no slanted connections between the layers are considered, as it may require different class of Green's functions [8]. In addition, although the algorithm developed in this paper is applied only to single-layer geometries, it can be extended to multilayered geometries with some modifications [23], which will be presented in the near future.

Since the subject matter of this paper is an efficient MoM-based analysis of printed circuits with multiple vertical strips, the MoM matrix entries corresponding to vertical strips require special attention. For such circuits, there are mainly five different inner-product terms involving $z$-directed basis and/or testing functions in the MoM matrix entries, which are $\left\langle T_{z i}, G_{z z}^{A} * B_{z j}\right\rangle,\left\langle\partial / \partial z T_{z i}, G_{z}^{q} * \partial / \partial z B_{z j}\right\rangle$ for $Z_{z z}^{i j},\left\langle\partial / \partial x T_{x i}, G_{z}^{q} * \partial / \partial z B_{z j}\right\rangle$ for $Z_{x z}^{i j}$, and $\left\langle T_{z i}, G_{z x}^{A} * B_{x j}\right\rangle$ and $\left\langle\partial / \partial z T_{z i}, G_{x}^{q} * \partial / \partial x B_{x j}\right\rangle$ for $Z_{z x}^{i j}$, where $B_{x j}, B_{z j}$ and $T_{x i}, T_{z i}$ denote the $j$ th basis and $i$ th testing functions used to expand the current density components in the $x$ - and $z$-directions, respectively, and $G_{z x}^{A}, G_{z z}^{A}, G_{x}^{q}$, and $G_{z}^{q}$ are the associated components of the Green's functions for vector and scalar potentials when the traditional form is used [8]. Since Green's functions-spectral-domain representations in planar multilayer media and their closed-form approximations in the spatial domain-have been detailed in the literature [24], they are not given here, except for the ones required in the formulation. One of the inner product terms given above, i.e., $\left\langle\partial / \partial z T_{z m}, G_{z}^{q} * \partial / \partial z B_{z n}\right\rangle$, which is the most difficult one as it involves integrations over both $z$ and $z^{\prime}$, is used to demonstrate the below formulation. Since the calculation of the other inner-product terms can be performed using similar steps, for the sake of brevity, they are not provided here. Using the definitions of inner-product and convolution integral, $\left\langle\partial / \partial z T_{z m}, G_{z}^{q} * \partial / \partial z B_{z n}\right\rangle$ can be written as

$$
\begin{aligned}
& \left\langle\frac{\partial}{\partial z} T_{z m}, G_{z}^{q} * \frac{\partial}{\partial z} B_{z n}\right\rangle \\
& =\iint d z d y \frac{\partial}{\partial z} T_{z m}(y, z) \\
& \quad \cdot \iint d z^{\prime} d y^{\prime} G_{z}^{q}\left(x-x^{\prime}, y-y^{\prime}, z, z^{\prime}\right) \frac{\partial}{\partial z^{\prime}} B_{z n}\left(y^{\prime}, z^{\prime}\right)
\end{aligned}
$$

where $x^{\prime}$ and $x$ denote the $x$-coordinates of the source and observation, respectively, both of which are constant at the vertical strips spanning in the $y$-direction. After some simple manipulations, (1) can be cast into the following form:

$$
\begin{aligned}
& \left\langle\frac{\partial}{\partial z} T_{z m}, G_{z}^{q} * \frac{\partial}{\partial z} B_{z n}\right\rangle \\
& =\iint d y d y^{\prime} T_{z m}(y) B_{z n}\left(y^{\prime}\right) \\
& \quad \cdot \int d z \frac{\partial}{\partial z} T_{z m}(z) \int d z^{\prime} G_{z}^{q}\left(|\boldsymbol{\rho}-\boldsymbol{\rho}|, z, z^{\prime}\right) \frac{\partial}{\partial z^{\prime}} B_{z n}\left(z^{\prime}\right)
\end{aligned}
$$

where the separability of the basis and testing functions is used. At first, the spatial-domain Green's function in (2) is written as the inverse Hankel transform of its spectral-domain representation

$$
\begin{aligned}
F_{z}^{q}(|\boldsymbol{\rho}-\boldsymbol{\rho}|) \stackrel{\text { def }}{=} & \iint d z d z^{\prime} \frac{\partial}{\partial z} T_{z m}(z) \\
& \cdot \frac{1}{4 \pi} \int_{S I P} d k_{\rho} k_{\rho} H_{0}^{(2)} \\
& \cdot\left(k_{\rho}|\boldsymbol{\rho}-\boldsymbol{\rho}|\right) \tilde{G}_{z}^{q}\left(k_{\rho} ; z, z^{\prime}\right) \frac{\partial}{\partial z^{\prime}} B_{z n}\left(z^{\prime}\right) \\
= & \frac{1}{4 \pi} \int_{S I P} d k_{\rho} k_{\rho} H_{0}^{(2)}\left(k_{\rho}|\boldsymbol{\rho}-\boldsymbol{\rho}|\right) \cdot \\
& \iint d z d z^{\prime} \frac{\partial}{\partial z} T_{z m}(z) \frac{\partial}{\partial z^{\prime}} B_{z n}\left(z^{\prime}\right) \\
& \cdot \tilde{G}_{z}^{q}\left(k_{\rho} ; z, z^{\prime}\right)
\end{aligned}
$$

where $\tilde{G}_{z}^{q}\left(k_{\rho} ; z, z^{\prime}\right)$ can be written analytically as an explicit function of $z$ and $z^{\prime}$ in the source layer as

$$
\begin{aligned}
\tilde{G}_{z}^{q}=\frac{1}{2 j k_{z_{i}} \epsilon_{i}}\{ & e^{-j k_{z_{i}}\left|z-z^{\prime}\right|}-\tilde{R}_{\mathrm{TM}}^{i, i+1} M_{\mathrm{TM}}^{i} e^{-j k_{z_{i}}\left(2 d_{i}-z^{\prime}-z\right)} \\
& -\tilde{R}_{\mathrm{TM}}^{i, i-1} M_{\mathrm{TM}}^{i} e^{-j k_{z_{i}}\left(z^{\prime}+z\right)} \\
& +\tilde{R}_{\mathrm{TM}}^{i, i+1} \tilde{R}_{\mathrm{TM}}^{i, i-1} M_{\mathrm{TM}}^{i} e^{-j k_{z_{i}}}\left(2 d_{i}+z^{\prime}-z\right) \\
& \left.+\tilde{R}_{\mathrm{TM}}^{i, i-1} \tilde{R}_{\mathrm{TM}}^{i, i+1} M_{\mathrm{TM}}^{i} e^{-j k_{z_{i}}\left(2 d_{i}+z-z^{\prime}\right)}\right\}
\end{aligned}
$$

and the generalized reflection coefficients $\tilde{R}_{\mathrm{TM}}^{i, i \pm 1}$ and other terms like $M_{\mathrm{TM}}^{i}$ can be found in [24]. Note that $\tilde{G}_{z}^{q}\left(k_{\rho} ; z, z^{\prime}\right)$ and the other Green's functions can be written as explicit functions of $z$ and $z^{\prime}$ not only in the source layer, but in any layer, which will be detailed in a future paper. After analytically evaluating $z$ and $z^{\prime}$ integrals in (3), $F_{z}^{q}$ can be obtained in closed form by the generalized pencil-of-function method [25] as a function of $\left|\rho-\rho^{\prime}\right|$. Hence, making the substitution $x-x^{\prime}=u$ and $y-y^{\prime}=v$, the inner-product term (2) reduces to

$$
\begin{aligned}
\left\langle\frac{\partial}{\partial z} T_{z m}, G_{z}^{q} * \frac{\partial B_{z n}}{\partial z}\right\rangle & \\
& =-\int d v F_{z}^{q}(u, v) \int d y T_{z m}(y) B_{z n}(y-v)
\end{aligned}
$$

where the inner integral can be evaluated analytically, and the resulting outer integral of $v$ can also be evaluated analytically following [12] or numerically. It should be pointed out that $F_{z}^{q}(u, v)$ is an analytic function of independent variables $u$ and $v$ explicitly. In obtaining $F_{z}^{q}$ in (3), the generalized pencil-offunction method is applied to the function obtained analytically from the inner double integral of (3). This integral-the term that the generalized pencil-of-function method is applied-can always be evaluated analytically because $z$ - and $z^{\prime}$-dependencies of spectral-domain Green's functions are always in exponential forms and, therefore, their products with most of the basis and testing functions and with their derivatives are analytically integrable over $z$ and $z^{\prime}$. Using the generalized pencil-offunction method for the resulting spectral-domain function in 
conjunction with the Sommerfeld identity, $F_{z}^{q}(u, v)$ is then obtained in closed form as a function of $u$ and $v$. Once $F_{z}^{q}(u, v)$ is obtained as such, it can be used for other vertical metallizations by just evaluating it for different $u$ and $v$ values provided that basis functions employed along the new vertical strips are the same as those used on the first strip. Therefore, adding new vertical strips would only require the evaluation of the outer integral in (5), whose computational cost is not significant, as it can be done analytically [12] or, perhaps more efficiently, numerically using an algorithm known as TRIEX (integration over a triangle using nonlinear extrapolation) [26].

Although the approach detailed above is quite general for any set of lengths of vertical conductors, the efficiency of the method for multiple vertical conductors can only be achieved when $z$ - and $z^{\prime}$-dependencies of the basis and testing functions for all vertical conductors are similar. To clarify this issue, one should refer to the analytic calculation of (3). In this equation, the generalized pencil-of-function method is applied to the integral of the product of the spectral-domain Green's function and $z$ - and $z^{\prime}$-derivatives of the testing and basis functions. If one decides to use an additional vertical strip with more basis functions (as opposed to two basis functions used throughout this study), then these basis functions and corresponding testing functions would have different $z$-dependencies, and would be integrated over different domains, resulting in different functions in (3) to be approximated by the generalized pencil-of-function method. Therefore, for every basis function with a different $z$-domain dependency, the generalized pencil-of-function method needs to be implemented separately for all inner-product terms involving these basis and/or corresponding testing functions. The same argument also applies to the other matrix entries involving $z$-directed basis and/or testing functions and, as a result, the matrix filling time increases in proportion to the number of basis functions with different $z$-domain representations or, in other words, in proportion to the number of applications of the generalized pencil-of-function method.

Before closing the discussion on the implementation of the proposed approach, a possible question as how to implement it efficiently in cases of multiple vertical strips with different lateral spans needs to be clarified. Remember that basis and testing functions used in this study are separable functions, and can be written in terms of two independent functions. Hence, using vertical strips with different spans does not pose a problem provided that their $z$-dependencies are the same. Once the $z$-dependencies are chosen to be identical, auxiliary functions can be obtained only once for every different $z$-domain representation of the basis or testing functions. As the auxiliary functions are obtained in closed form after the exponential approximation, they can be used repeatedly in the evaluations of the matrix entries corresponding to testing and/or basis functions with the same $z$-and/or $z^{\prime}$-dependencies. Considering the inner-product term (1) whose evaluation has been detailed above, it does not matter if the strip spans on $x$ - or $y$-directions since only the $z$-dependencies of the testing and basis functions are involved in its evaluation. Therefore, for any additional vertical strip (regardless of its direction of span) with a different location on the $x y$-plane, but with the same $z$ and $z^{\prime}$ representation, the same auxiliary function is to be used with different integration limits over $x, y, u$, and $v$.

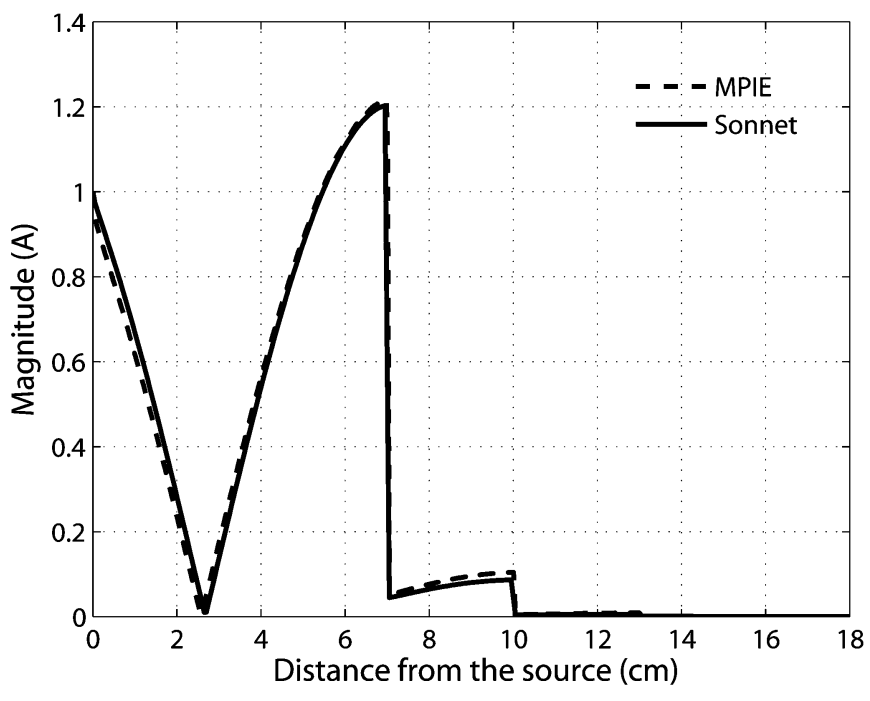

Fig. 2. Current distributions along the microstrip-line circuit, shown in Fig. 1, at $f=1.0 \mathrm{GHz}$.

\section{B. Demonstration}

Once the formulation is set up, it would be instructive to see how it is working on a simple and intuitive example, such as a microstrip line with multiple shorting strips (Fig. 1). The widths of the microstrip line and vertical strips are chosen to be narrow to facilitate the use of only longitudinal current density $J_{x}(x, y)$ on the microstrip line for the sake of simplicity. Since the thicknesses of substrates used in microwave circuits and printed antennas are usually small, as compared to wavelength, two basis functions would be sufficient to represent the current density $J_{z}(y, z)$ on each vertical strip. In cases of thicker substrates, one can model the substrate as composed of two or more layers with identical electrical properties, resulting in as many basis functions as needed along the vertical conductors. Using the same number of basis functions on every vertical strip guarantees that the basis functions on each strip will have identical $z$-variations, which is the main requirement for the efficiency of the method, as discussed above.

At first, to validate the proposed method, the current distribution along the microstrip line is obtained and compared to that obtained from a commercial software, em by SONNET Software, North Syracuse, NY, in Fig. 2. It is observed that the current distributions agree quite well. Once the method is validated, the efficiency of the method is assessed by studying the computational cost of filling the MoM matrix for each additional vertical strip. In this study, it is assumed that the MoM matrix entries corresponding to the horizontal part of the geometry are filled once and not calculated again with the additional vertical strips. Therefore, counting new MoM matrix entries, and their computational expensive constituents, corresponding to additional vertical strips would be sufficient to assess the efficiency of the method. The filling time of MoM matrix entries corresponding to each additional vertical strip is recorded for a microstrip line with $N=71$, as shown in Table I, for two different scenarios: using the information of identical $z$-variations of the basis functions along vertical strips as proposed in this paper (case 1) and not using any information to fill the matrix, except 
TABLE I

MATRIX Fill Times FOR EACH AdDitional VerTiCAL STRIP FOR THE GEOMETRY IN FIG. $1, N=71$

\begin{tabular}{|c|c|c|}
\hline \multirow{2}{*}{ Number of vertical strips } & \multicolumn{2}{|l|}{$\begin{array}{l}\text { MoM matrix fill-time for } \\
\text { additional entries }\end{array}$} \\
\cline { 2 - 3 } & Case I (sec) & Case II (sec) \\
\hline 1 & 11.8 & 69.8 \\
\hline 2 & 4.0 & $68.6^{\mathrm{a}}$ \\
\hline 3 & 4.1 & 72.2 \\
\hline 4 & 4.2 & 75.8 \\
\hline
\end{tabular}

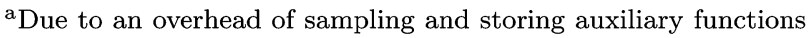
only once for the numerical integrations of the inner-products during the inclusion of the first strip, adding the second strip appears to be less time-consuming.

for the symmetry of the Galerkin's implementation of the MoM procedure (case 2). In the former scenario, the necessary auxiliary functions need to be calculated only once and are used repeatedly, but for the latter, the auxiliary functions are calculated for every inner-product term corresponding to each basis and testing functions introduced with the addition of a new vertical strip. Note that a slight decrease in the computational cost of adding a second vertical strip, as compared to that of the first one (Case II in Table I) is due to the implementation of the numerical integration algorithm, requiring the samples of auxiliary functions only once. It is clearly observed from Table I that once one vertical strip is introduced into the formulation and the MoM matrix is filled, adding new vertical strips does not require any significant additional computational cost. To understand this, let us investigate the additional matrix entries and their constituents.

The number of additional rows and columns for the MoM matrix, when a vertical strip is added, is equal to the number of basis functions on the strip. Since it was already noted that two basis functions on each vertical strip are employed, two rows and columns are to be filled in and included in the MoM matrix. However, if the Galerkin's MoM is used, only the columns or rows would be sufficient, due to the symmetry of the MoM matrix. For these new entries, there are generally five inner-product terms to be evaluated for each distinct basis and testing functions, given as

$$
\begin{aligned}
& \left\langle T_{z}, G_{z z}^{A} * B_{z}\right\rangle \text { and }\left\langle\frac{\partial}{\partial z} T_{z}, G_{z}^{q} * \frac{\partial}{\partial z} B_{z}\right\rangle \text { for } Z_{z z} \\
& \left\langle\frac{\partial}{\partial x} T_{x}, G_{z}^{q} * \frac{\partial}{\partial z} B_{z}\right\rangle \text { for } Z_{x z} \\
& \left\langle T_{z}, G_{z x}^{A} * B_{x}\right\rangle \text { and }\left\langle\frac{\partial}{\partial z} T_{z}, G_{x}^{q} * \frac{\partial}{\partial x} B_{x}\right\rangle \text { for } Z_{z x}
\end{aligned}
$$

however, for the Galerkin's MoM, the terms for $Z_{z z}$ and $Z_{x z}$ would be sufficient. Since they involve integrals and/or derivatives on $z$ and/or $z^{\prime}$ variables, the closed-form auxiliary functions are used instead of closed-form Green's functions in the evaluations of these inner-product terms. As the generalized pencil-of-function method is the main time-consuming numerical block of the computation of the auxiliary functions, as discussed in Section II-A, the number of generalized pencil-of-

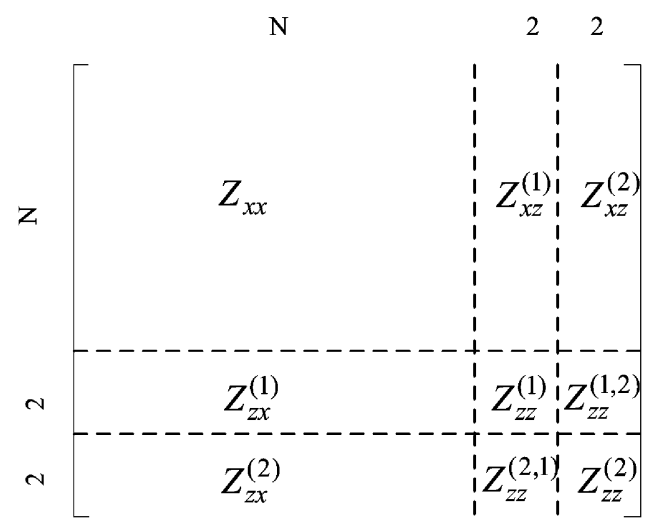

Fig. 3. MoM matrix entries for Fig. 1 with two vertical strips.

function implementation per vertical strip can be used as a measure of efficiency. To facilitate counting the number of generalized pencil-of-function implementation, it should be noted that evaluation of each auxiliary function requires three generalized pencil-of-function implementations, as a three-level algorithm is used for the auxiliary functions and a two-level algorithm is used for the Green's functions. Therefore, adding an $n$th vertical strip to already available $(n-1)$ vertical strips requires the following counts of auxiliary function evaluations for the Galerkin's MoM:

$$
\underbrace{(3 \times 2)}_{Z_{z z}^{(n)}}+(n-1) \underbrace{(3 \times 2)}_{Z_{z z}^{(m, n)}}+\underbrace{(N \times 2)}_{Z_{x z}^{n}}+\underbrace{(n \times 2)}_{\text {Attachment }}
$$

where $N$ is the number of basis function for the horizontal part of the geometry, as the partitions of the MoM matrix and their dimensions are shown in Fig. 3. Note that the counts in (6) have been obtained with the following assumptions.

1) The matrix is symmetric as the Galerkin's MoM is used.

2) Two basis functions are employed to represent the current density along each vertical strip.

3) Attachment basis functions are introduced at the junctions of horizontal and vertical conductors to satisfy the current continuity and charge conservation.

4) Auxiliary functions are recalculated for each different term, as if the MoM matrix is being filled using a standard MoM approach.

To get an idea as to how the above counts are obtained for the Galerkin's MoM, here are some numbers and their origins: for the first vertical strip, the number of auxiliary function evaluations is $(3 \times 2)+(N \times 2)+(2 \times 1)$, where $(3 \times 2)$ corresponds to three different elements in $Z_{z z}^{(1)}$ with two inner-product terms in each, $(N \times 2)$ denotes the number of entries in $Z_{x z}^{(1)}$ with one inner-product term, and $(2 \times 1)$ auxiliary functions account for the attachment function contribution with one inner-product term; for the second vertical strip, $(3 \times 2)+(3 \times 2)+(N \times$ $2)+(2 \times 2)$ gives the additional number of auxiliary function evaluations, where the second term is due to the different entries in $Z_{z z}^{(1,2)}$.

Since the formula in (6) and the numbers given above provide the number of auxiliary function evaluations for the $n$th additional vertical strip, i.e., incremental change, the total number 


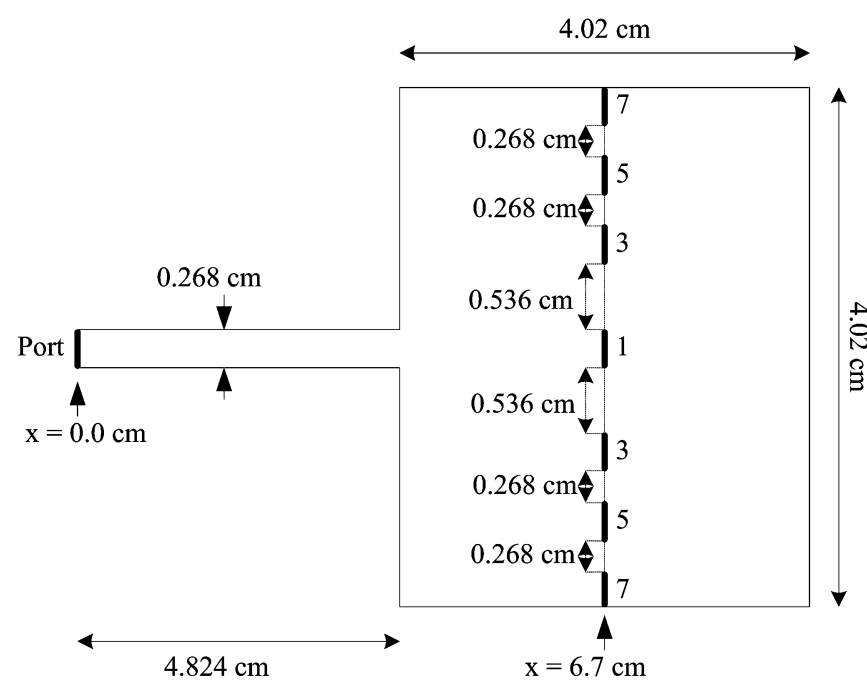

Fig. 4. Geometry of a patch antenna using multiple vertical strips.

of auxiliary function evaluations for $M$ additional vertical strips to a horizontal geometry, in the case of Galerkin's MoM, can be easily calculated from (6) as $3 M(M+1)+M(N \times 2)+M(M+$ $1)$. Thus, to assess the computational burden with adding $M$ vertical strips to a horizontal geometry, the following example represents the case provided in Fig. 1 for two different discretizations: for $N=71$, adding four new vertical strips requires 648 auxiliary function evaluations and $648 \times 3$ generalized pencil-of-function implementations; for $N=359$, it requires 2952 auxiliary function evaluations and $2952 \times 3$ generalized pencil-of-function implementations. However, with the use of the proposed method, assuming that all vertical strips have the same length and the same number of basis functions (two in this case), $(3 \times 2)+2$ auxiliary function evaluations will be sufficient for any number of vertical conductors, i.e., independent of the number of vertical strips. Note that $(3 \times 2)$ is for three different elements in $Z_{z z}^{(1)}$ with two inner-product terms each, and 2 is for two different basis functions with different $z^{\prime}$ variations out of $(N \times 2)$ elements of $Z_{x z}^{(1)}$ with one inner-product term each. Note that, as attachment functions used with each vertical strip are defined over the planar conductors, inner-products involving them do not require any auxiliary function evaluations.

\section{NUMERICAL EXAMPLES}

Thus far, an electromagnetic-based simulation algorithm was introduced together with its demonstration on a simple example with thorough discussions on its efficiency when multiple vertical strips were involved. Here, the proposed algorithm is applied to more practical printed circuits and patch antennas, and their $S$-parameters are presented and, if available, are compared to experimental results in the literature.

To start with, let us consider a patch antenna whose geometry and dimensions are given in Fig. 4 where the thickness $h$ and dielectric constant $\epsilon_{r}$ of the substrate are set to $0.132 \mathrm{~cm}$ and 2.33, respectively. Note that vertical strips employed in this study are chosen to be spanning in the $y$-direction with the same

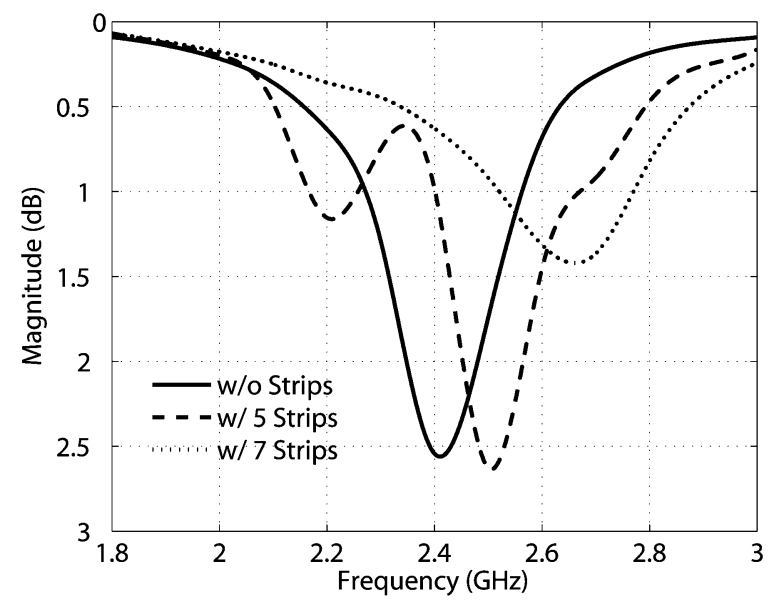

Fig. 5. Magnitudes of $S_{11}$ for the patch in Fig. 4, with zero, five, and seven shorting strips.

width of $0.268 \mathrm{~cm}$, and all are located at $x=6.7 \mathrm{~cm}$ to approximate short circuiting the patch at a distance of $1.876 \mathrm{~cm}$ from its left edge. All vertical strips have the same length (equals to the thickness of the substrate) as they are short circuiting the patch to ground and, therefore, the basis and testing functions have the same $z$ - and $z^{\prime}$-dependencies, enabling the algorithm to efficiently analyze the effects of multiple vertical strips. The choice of a patch antenna with vertical strips, as an example, is motivated by the facts that: 1 ) vertical strips are used to achieve multifunction antennas, like dual-band dual-polarized antennas and 2) the results expected and achieved could be interpreted intuitively, as the patch would be expected to have resonances (according to the cavity model) at the frequencies of $2.48 \mathrm{GHz}$ $\left[=c /\left(2 \times 4.02 \times \sqrt{\epsilon_{\mathrm{eff}}}\right)\right]$ and $2.65 \mathrm{GHz}\left[=c /\left(4 \times 1.876 \times \sqrt{\epsilon_{\mathrm{eff}}}\right)\right]$ with no shorting strip and with full short circuiting at a distance of $1.876 \mathrm{~cm}$ from the feeding edge, respectively, where $\epsilon_{\mathrm{eff}}$ is calculated heuristically from [27]. As the first step, the implementation of the algorithm requires meshing the horizontal and vertical conductors in the geometry: the square patch is meshed into $15 \times 15$ cells, employing $420(=2 \times 15 \times 14)$ basis functions for the current densities on the patch $J_{x}$ and $J_{y}$ together, 18 basis functions for the feeding line, and two basis functions for each vertical strip. Once the algorithm is applied to the geometry depicted in Fig. 4, with the necessary information as provided above, $S_{11}$ is obtained from the current density over a band of frequency. To observe the effect of the shorting strips, the geometry is first analyzed with zero, one, and three vertical strips, and then strips with numbers 5 and 7 are added consecutively, where the numbers appear just next to the strips in Fig. 4. The magnitudes of $S_{11}$ are provided in Fig. 5, and the resonant frequencies of the patch with no vertical strip and with seven vertical strips are observed to be approximately 2.42 and $2.65 \mathrm{GHz}$, which are in good agreement with the above predicted resonant frequencies.

Since the main objective here is to demonstrate the efficiency of the method rather than characterization of different structures, the matrix-fill time for the entries corresponding to each additional vertical strip is studied and provided in Table II. Note that using the procedure developed in this paper requires almost 
TABLE II

MATRIX FILl TIMES FOR EACH AdDITIONAL VeRTICAL STRIP FOR THE GEOMETRY IN FIG. $4, N=438$

\begin{tabular}{|c|c|c|}
\hline \multirow{2}{*}{ Number of vertical strips } & \multicolumn{2}{|c|}{$\begin{array}{l}\text { MoM matrix fill-time for ad- } \\
\text { ditional entries }\end{array}$} \\
\cline { 2 - 3 } & Case I (sec) & Case II (sec) \\
\hline 1 & 23.8 & 435.5 \\
\hline 2 & 18.7 & 437.7 \\
\hline 3 & 18.8 & 441.4 \\
\hline$\vdots$ & $\vdots$ & $\vdots$ \\
\hline 7 & 19.0 & 456.2 \\
\hline
\end{tabular}

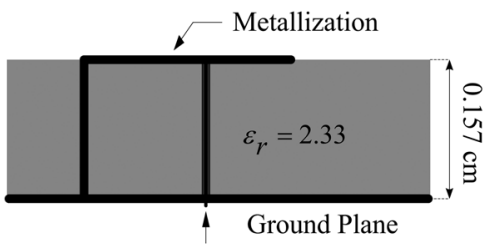

Coaxial Feed

(a)

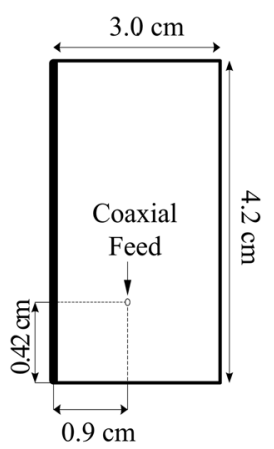

(b)
Fig. 6. Single layered patch antenna. (a) Side view. (b) Top view.

half a minute for the first vertical strip and even less for the additional vertical strips when no symmetry argument of the geometry is used to further reduce the time. It is clear that the computational cost of an additional vertical strip, in the case of a uniformly meshed patch antenna using the symmetry of the geometry, would be on the order of a few seconds. However, for a general-purpose algorithm that employs nonuniform meshing, the algorithm proposed in this paper reduces the cost of computation significantly, an order of magnitude, rendering the algorithm extremely suitable for optimization purposes.

As a final example, a planar inverted "F" antenna (PIFA), which is a rectangular patch antenna with one side short circuited to ground using multiple vertical strips, is studied. This geometry is a modified version of a PIFA studied in [20] using a whole wide shorting plate instead of nontouching vertical strips, as shown in Fig. 6. First, the input impedance is obtained for 15 nontouching narrow vertical strips over a band of frequency, and the results are compared to those provided in [20] for the same geometry, except for the implementation of the shorting plate. Although the loci of the input impedance curves match quite well, the input impedances for each frequency differ, as this would be expected due to a different kind of loading at the left-edge of the patch antenna (Fig. 7). In addition to mimicking a shorting plate in a PIFA, using nontouching vertical strips may provide an additional degree of freedom in the design of such antennas with different specifications when combined with an efficient CAD tool, as proposed in this paper. For the sake of completeness, the matrix-fill time for the entries corresponding to each additional vertical strip is provided in Table III, where it is clearly observed that using the algorithm proposed in this

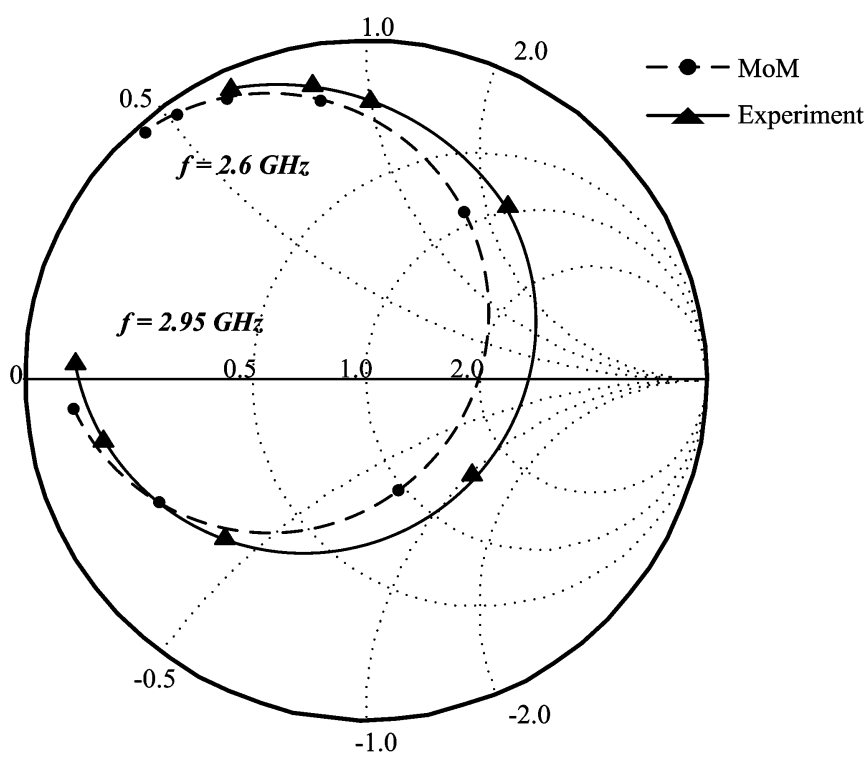

Fig. 7. Input impedance of the antenna shown in Fig. 6.

TABLE III

Matrix Fill Times for EACH AdDitional Vertical StRIP FOR THE GEOMETRY IN FIG. $6, N=565$

\begin{tabular}{|c|c|c|}
\hline \multirow{2}{*}{ Number of vertical strips } & \multicolumn{2}{|c|}{$\begin{array}{l}\text { MoM matrix fill-time for ad- } \\
\text { ditional entries }\end{array}$} \\
\cline { 2 - 3 } & Case I (sec) & Case II (sec) \\
\hline 1 (Feed) & 28.9 & 424.4 \\
\hline 2 & 18.6 & 420.9 \\
\hline 3 & 18.9 & 424.6 \\
\hline$\vdots$ & $\vdots$ & $\vdots$ \\
\hline 16 & 22.8 & 472.7 \\
\hline
\end{tabular}

paper significantly improves the computation time when compared to straightforward use of the combination of the MoM and the discrete complex image method.

\section{CONCLUSIONS}

In this study, a computationally efficient algorithm, based on the combination of the MoM and the discrete complex image method, has been proposed and demonstrated for the analysis of printed geometries with multiple vertical conductors. The main contribution is that the proposed algorithm can handle multiple vertical conductors extremely efficiently, i.e., the computational cost of additional vertical conductors is improved almost an order of magnitude when compared to a straightforward implementation of the very same method. Although the method is presented for single-layer geometries, it can handle vertical conductors extending over multiple layers in a multilayer environment with some modifications, whose detail can be found in [23], and will be submitted for future publication soon. In conclusion, the algorithm proposed and demonstrated in this paper is extremely efficient and may be one of the best candidates for an efficient CAD simulation software that can combine any optimization method for the design of microwave circuits and microstrip antennas involving multiple vertical conductors. 


\section{REFERENCES}

[1] J. R. James and G. Andrasic, "Multifunction printed antennas," in $A d-$ vances in Microstrip and Printed Antennas, K. F. Lee and W. Chen, Eds. New York: Wiley, 1997.

[2] S. Shahparnia and O. M. Ramahi, "Electromagnetic interference (EMI) reduction from printed circuit boards $(\mathrm{PCB})$ using electromagnetic bandgap structures," IEEE Trans. Electromagn. Compat., vol. 46, no. 4, pp. 580-587, Nov. 2004.

[3] C. Wang, M. Leone, J. L. Leone, A. L. Drewniak, and A. Orlandi, "Coupling between differential signals and the DC power-bus in multilayer PCBs," IEEE Trans. Adv. Packag., vol. 28, no. 2, pp. 337-345, May 2005.

[4] N. Herscovici, "New considerations in the design of microstrip antennas," IEEE Trans. Antennas Propag., vol. 46, no. 6, pp. 807-812, Jun. 1998.

[5] M. J. Tsai, F. D. Flavis, O. Fordharn, and N. G. Alexopoulos, "Modeling planar arbitrarily shaped microstrip elements in multilayered media," IEEE Trans. Microw. Theory Tech., vol. 45, no. 3, pp. 330-337, Mar. 1997.

[6] M. R. Abdul-Gaffoor, H. K. Smith, A. A. Kishk, and A. W. Glisson, "Simple and efficient full-wave modeling of electromagnetic coupling in realistic RF multilayer PCB layouts," IEEE Trans. Antennas Propag., vol. 50, no. 6, pp. 1445-1457, Jun. 2002.

[7] J. R. Mosig, "Arbitrarily shaped microstrip structures and their analysis with a mixed potential integral equation," IEEE Trans. Microw. Theory Tech., vol. 36, no. 2, pp. 314-323, Feb. 1988.

[8] K. A. Michalski and D. Zheng, "Electromagnetic scattering and radiation by surfaces of arbitrary shape in layered media, part 1: Theory," IEEE Trans. Antennas Propag., vol. 38, no. 3, pp. 335-344, Mar. 1990.

[9] D. G. Fang, J. J. Yang, and G. Y. Delisle, "Discrete image theory for horizontal electric dipoles in a multilayered medium," Proc. Inst. Elect. Eng., vol. 135 , no. 5, pt. H, pp. 297-303, Oct. 1988.

[10] Y. L. Chow, J. J. Yang, D. G. Fang, and G. E. Howard, "A closed-form spatial Green's function for the thick microstrip substrate," IEEE Trans. Microw. Theory Tech., vol. 39, no. 3, pp. 588-592, Mar. 1991.

[11] M. I. Aksun, "A robust approach for the derivation of closed-form Green's functions," IEEE Trans. Microw. Theory Tech., vol. 44, no. 5, pp. 651-658, May 1996.

[12] L. Alatan, M. I. Aksun, K. Mahadevan, and T. Birand, "Analytical evaluation of the MoM matrix elements," IEEE Trans. Microw. Theory Tech., vol. 44, no. 4, pp. 519-525, Apr. 1996.

[13] K. Noyan and M. I. Aksun, EMPLAN: Electromagnetic Analysis of Printed Structures in Planarly Layered Media. Norwood, MA: Artech House, 2000.

[14] M. I. Aksun and R. Mittra, "Efficient use of closed-form Green's functions for three-dimensional problems involving multilayered media," in IEEE AP-S Int. Symp. Dig., Jun. 1994, vol. 2, pp. 1354-1357.

[15] K. A. Michalski and J. R. Mosig, "Discrete complex image MPIE analysis of coax-fed coupled vertical monopoles in grounded dielectric substrate: Two formulations," Proc. Inst. Elect. Eng.-Microw., Antennas, Propag., vol. 142, no. 3, pp. 269-274, Jun. 1995.

[16] _ "Discrete complex image mixed-potential integral equation analysis of microstrip patch antennas with vertical probe feeds," Electromagnetics, vol. 15, pp. 377-392, Jul./Aug. 1995.

[17] N. Kinayman and M. I. Aksun, "Efficient use of closed-form Green's functions for the analysis of planar geometries with vertical connections," IEEE Trans. Microw. Theory Tech., vol. 45, no. 5, pp. 593-603, May 1997.

[18] Y. Liu, L.-W. Li, T.-S. Yeo, and M.-S. Leong, "Application of DCIM to MPIE-MoM analysis of 3-D PEC objects in multilayered media," IEEE Trans. Antennas Propag., vol. 50, no. 2, pp. 157-162, Feb. 2002.

[19] F. Ling, J. Liu, and J.-M. Jin, "Efficient electromagnetic modeling of three-dimensional multilayer microstrip antennas and circuits," IEEE Trans. Microw. Theory Tech., vol. 50, no. 6, pp. 1628-1635, Jun. 2002.

[20] T. M. Grzegorczyk and J. R. Mosig, "Full-wave analysis of antennas containing horizontal and vertical metallizations embedded in planar multilayered media," IEEE Trans. Antennas Propag., vol. 51, no. 11, pp. 3047-3054, Nov. 2003.

[21] M. Vrancken and G. A. E. Vandenbosch, "Hybrid dyadic-mixed-potential and combined spectral-space domain integral-equation analysis of quasi-3-D structures in stratified media," IEEE Trans. Microw. Theory Tech., vol. 51, no. 1, pp. 216-224, Jan. 2003.
[22] K. Naishadham and P. Misra, "Order recursive Gaussian elimination and efficient CAD of microwave circuits," in IEEE MTT-S Int. Microw. Symp. Dig., Orlando, FL, May 16-20, 1995, vol. 3, pp. 1435-1438.

[23] T. Onal, "Development of a rigorous and efficient electromagnetic simulation algorithm for 3-D printed structures in multilayer environment,' M.S. thesis, Dept. Elect. Comput. Eng., Koç Univ., Istanbul, Turkey, 2005.

[24] G. Dural and M. I. Aksun, "Closed-form Green's functions for general sources and stratified media," IEEE Trans. Microw. Theory Tech., vol. 43, no. 7, pp. 1545-1552, Jul. 1995

[25] H. Yingbo and T. K. Sarkar, "Generalized pencil-of-function method for extracting poles of an EM system from its transient response," IEEE Trans. Antennas Propag., vol. 37, no. 2, pp. 229-234, Feb. 1989.

[26] E. D. Doncker and I. Robinson, "TRIEX: Integration over a triangle using nonlinear extrapolation," ACM Trans. Math. Softw., vol. 10, pp. 17-22, Mar. 1984.

[27] K. P. Ray and G. Kumar, "Determination of the resonant frequency of microstrip antennas," Microw. Opt. Technol. Lett., vol. 23, pp. 114-117, Oct. 1999.

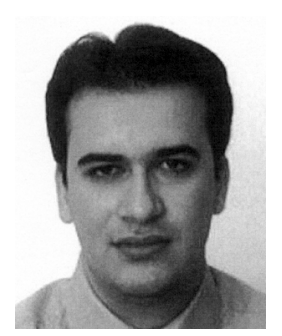

Tayyar Onal was born in Konya, Turkey, in 1982. He received the B.S. degree in electrical and electronics engineering from Bilkent University, Ankara, Turkey, in 2003, the M.S. degree in electrical and electronics engineering from Koç University, Istanbul, Turkey, in 2005 , and is currently working toward the Ph.D. degree in electrical and electronics engineering at Koç University.

His research interests includes numerical analysis of printed structures, microwave and millimeter-wave circuits, and microstrip antennas.

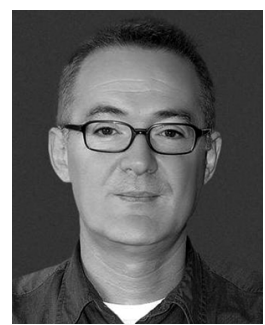

M. I. Aksun (M'92-SM'99) received the B.S. and M.S. degrees in electrical and electronics engineering from Middle East Technical University, Ankara, Turkey, in 1981 and 1983, respectively, and the Ph.D. degree in electrical and computer engineering from the University of Illinois at Urbana-Champaign, in 1990.

From 1990 to 1992, he was a Post-Doctoral Fellow with the Electromagnetic Communication Laboratory, University of Illinois at Urbana-Champaign. From 1992 to 2001, he was a member of the faculty of the Department of Electrical and Electronics Engineering, Bilkent University, Ankara, Turkey, and became a Professor in 1999. In 2001, he has joined the Department of Electrical and Electronics Engineering, Koç University, Istanbul, Turkey, as a Professor. In May 2004, he became the Dean of the Engineering College, Koç University. His research interests include numerical methods for electromagnetics and optics, printed circuits and antennas, and indoor and outdoor propagation models.

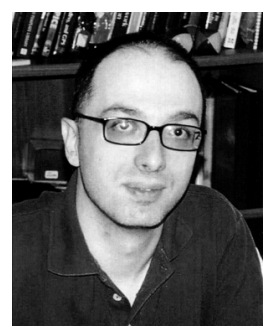

Noyan Kinayman (S'94-M'96) received the B.S. and M.S. degrees from Middle East Technical University, Ankara, Turkey, in 1990 and 1993 , respectively, and the Ph.D. degree from Bilkent University, Ankara, Turkey, in 1997, all in electrical engineering.

Upon completion of his doctoral studies, he joined the Corporate Research and Development Department, M/A-COM, Lowell, MA, as Senior Electrical Engineer. He is currently with the same department as a Principal Electrical Engineer. His main responsibilities are electromagnetic analysis, modeling, and microwave circuit design. He has developed commercially available full-wave electromagnetic simulation software to simulate planar microstrip circuits. His main professional interests are electromagnetic theory, numerical solution of electromagnetic problems, and model extraction of passive microwave printed circuits. 